\title{
Measurement of intrinsic properties of amyloid fibrils by the peak force QNM method
}

\author{
Jozef Adamcik, ${ }^{a}$ Cecile Lara, ${ }^{a}$ Ivan Usov, ${ }^{a}$ Jae Sun Jeong, ${ }^{b}$ Francesco S. Ruggeri, ${ }^{b}$ Giovanni Dietler, ${ }^{b}$ \\ Hilal A. Lashuel, ${ }^{c}$ Ian W. Hamley ${ }^{d}$ and Raffaele Mezzenga ${ }^{* a}$
}

Received 30th March 2012, Accepted 9th May 2012

DOI: 10.1039/c2nr30768e

\begin{abstract}
We report the investigation of the mechanical properties of different types of amyloid fibrils by the peak force quantitative nanomechanical (PF-QNM) technique. We demonstrate that this technique correctly measures the Young's modulus independent of the polymorphic state and the cross-sectional structural details of the fibrils, and we show that values for amyloid fibrils assembled from heptapeptides, $\alpha$-synuclein, $\mathbf{A} \beta(\mathbf{1}-42)$, insulin, $\beta$-lactoglobulin, lysozyme, ovalbumin, Tau protein and bovine serum albumin all fall in the range of 2-4 GPa.
\end{abstract}

\section{Introduction}

The study of the self-assembly of peptides and unfolded proteins into highly ordered fibrillar nanostructures, referred to as "amyloid fibrils" and characterised by a dominant $\beta$-sheet secondary structure, is a quickly growing field due to its relevance in biology, medicine, biophysics, and materials science. The main source of interest in these systems is due to their association with a number of neurodegenerative and pathological human diseases such as Parkinson's, Alzheimer's, Creutzfeldt-Jakob diseases and type II diabetes. ${ }^{1-5}$ However, occurrence of amyloid fibrils with normal biological activities unrelated to diseases is also found in nature: these systems are referred to as "functional amyloid fibrils". $6-8$ In addition, the ability of a wide range of disease-unrelated proteins and artificial peptides to form amyloid-like structures in vitro makes amyloid fibrils very good candidates in food, biomedical, and nanotechnology

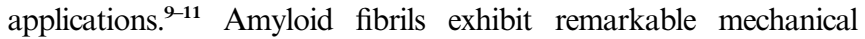
properties with values of Young's moduli in the order of several GPa due to the strong ordering of hydrogen bonds between the $\beta$ sheets. ${ }^{11-13}$ For instance, insulin has been shown to possess a Young's modulus of $3.3 \pm 0.4 \mathrm{GPa}^{13}$

${ }^{a}$ ETH Zurich, Food \& Soft Materials Science, Institute of Food, Nutrition \& Health, Schmelzbergstrasse 9, LFO E23, 8092 Zurich, Switzerland. E-mail: raffaele.mezzenga@hest.ethz.ch; Fax: +41 4463216 03; Tel: +41446329140

${ }^{b}$ Laboratory of Physics of Living Matter, Ecole Polytechnique Fédérale de Lausanne, CH-1015 Lausanne, Switzerland

'Laboratory of Molecular and Chemical Biology of Neurodegeneration, Brain Mind Institute, School of Life Sciences, Ecole Polytechnique Fédérale de Lausanne, CH-1015 Lausanne, Switzerland

${ }^{d}$ Dept of Chemistry, University of Reading, Whiteknights Reading, RG6 $6 A D, U K$
The assessment of mechanical and structural properties at the single fibril level provides very important information for the understanding of the role of amyloid fibrils in a biological context, and can allow tuning their functionality in nanomaterials applications. For example, with respect to the biological role of amyloid fibrils, it is now established that fibril fragmentation can enhance cytotoxicity. ${ }^{14}$ To this end, both experimental and theoretical approaches have been considered. Atomic force microscopy (AFM) represents one of the most suitable experimental techniques for quantitative measurements of local elasticity of fibrils, due to the possibility of imaging at the single molecule level ${ }^{12,15,16}$ and imposing and measuring forces down to the piconewton range, ${ }^{13,17}$ although indirect procedures are typically required to extract intrinsic mechanical properties. On the other hand, estimations of Young's moduli of amyloid fibrils by simulations point at numbers in the same order of magnitude as experimental values, yet, systematically higher. ${ }^{18-21}$

Recently, we have reported that peak force quantitative nanomechanical (PF-QNM) AFM shows great potential to be applied as a high-resolution technique to identify structural features and associated nanomechanical properties of amyloid fibrils. ${ }^{22}$

Here we further demonstrate that the technique is insensitive to the structural details of the amyloid fibril cross-section, that is to say that the intrinsic stiffness expressed by Young's modulus can be correctly de-coupled from the overall rigidity, to which both Young's modulus and cross-section contribute. We then apply the technique to different classes of amyloid fibrils, to measure the Young's modulus for an end-capped heptapeptide, $\alpha$-synuclein, $\mathrm{A} \beta(1-42)$, Tau protein, insulin, $\beta$-lactoglobulin, lysozyme, ovalbumin and bovine serum albumin fibrils and we show that all values of Young's moduli are in the range of $2-4 \mathrm{GPa}$.

\section{Materials and methods}

\section{Amyloid fibrils preparation}

All the amyloid fibrils were produced in vitro according to established protocols. Below, we give only a short experimental summary, addressing the reader to individual published procedures for more details. The peptide $\mathrm{CH}_{3} \mathrm{CONH}-\beta \mathrm{A} \beta \mathrm{AKLVFF}-\mathrm{CONH}_{2} \quad\left(M_{\mathrm{w}}\right.$ $835.93 \mathrm{Da}$ ) was custom-synthesized by C.S. Bio Company (USA) and used as received, without further purification and $0.5 \mathrm{wt} \%$ solution was prepared by dilution of the peptide in Milli-Q water. ${ }^{23,24}$ 
BioPURE- $\beta$-lactoglobulin ${ }^{25}$ was obtained from Davisco Foods International, Inc. (Le Sueur, MN). Hen egg white lysozyme, ${ }^{26,27}$ ovalbumin from chicken egg white, bovine serum albumin and insulin were purchased from Sigma. All proteins were dialyzed for one week at $4{ }^{\circ} \mathrm{C}$ against $\mathrm{pH} 2$ Milli-Q water and freeze-dried. $2 \mathrm{wt} \%$ protein solutions, prepared in Milli-Q water at $\mathrm{pH} 2$, were placed in an oil bath at $90^{\circ} \mathrm{C}$ and incubated with magnetic agitation (300 rpm).

Aliquots were taken at the given times ranging from 1 to $265 \mathrm{~h}$, quenched in ice-cold water and stored at $4{ }^{\circ} \mathrm{C}$. A $\beta(1-42),{ }^{28} \alpha$-synuclein, ${ }^{29}$ and tau ${ }^{30}$ fibrils were grown at $37^{\circ} \mathrm{C}$ in physiological buffers.

\section{Sample preparation}

All solutions containing amyloid fibrils were diluted to a final protein concentration of $0.1 \mathrm{wt} \%$ in $\mathrm{pH} 2$ Milli-Q water. A $20 \mu \mathrm{L}$ aliquot of each solution was deposited onto freshly cleaved mica, in case of $\mathrm{A} \beta(1-42)$ on modified mica, ${ }^{31}$ incubated for 2 minutes, rinsed with Milli-Q water and dried by air.

\section{PF-QNM experiments}

PF-QNM measurements were performed by using a MultiMode VIII Scanning Probe Microscope (Bruker, USA) operated in intermittent mode under ambient conditions at a scan rate of $1 \mathrm{~Hz}$. The microscope was covered with an acoustic hood to minimize vibrational noise. The AFM cantilevers (Bruker, USA) were calibrated on the calibration samples (Bruker, USA) - typically low-density polyethylene and polystyrene - covering the following ranges of Young's moduli: from $100 \mathrm{MPa}$ to $2 \mathrm{GPa}$ (for low-density polyethylene) and from 1 to $20 \mathrm{GPa}$ (for polystyrene). ${ }^{22}$ The analysis of the Derjaguin-Mueller-Toporov (DMT) modulus was performed by the software Nanoscope Analysis.

\section{Results and discussion}

The overall rigidity or flexibility of amyloid fibrils is well described by the persistence length $l_{\mathrm{p}}$, which is the typical length at which thermal fluctuations begin to bend the fibril in different directions. The persistence length encodes the intrinsic stiffness of the fibril and the geometry of its cross-section, correlated by the relation $l_{\mathrm{p}}=E I / k_{\mathrm{B}} T$, where $E$ is the Young's modulus of the fibril, $I$ is the area moment of inertia of the fibril cross-section, $k_{\mathrm{B}}$ is the Boltzmann constant and $T$ is the temperature.

Amyloid fibrils are often composed of several protofilaments. Therefore the area moment of inertia $I$ depends specifically on the packing geometry of individual protofilaments and the number of protofilaments in mature fibrils. ${ }^{32}$ When the exact packing scheme of the different protofilaments within the same amyloid fibril is established and the value of the persistence length can be measured, the precise estimation of the Young's modulus of the amyloid fibril can be determined. This approach has been successfully employed to determine the Young's modulus of $\beta$-lactoglobulin fibrils, for which the packing schemes of the protofilaments are now well-established. ${ }^{15}$ A first success of the PF-QNM method has been to show that the
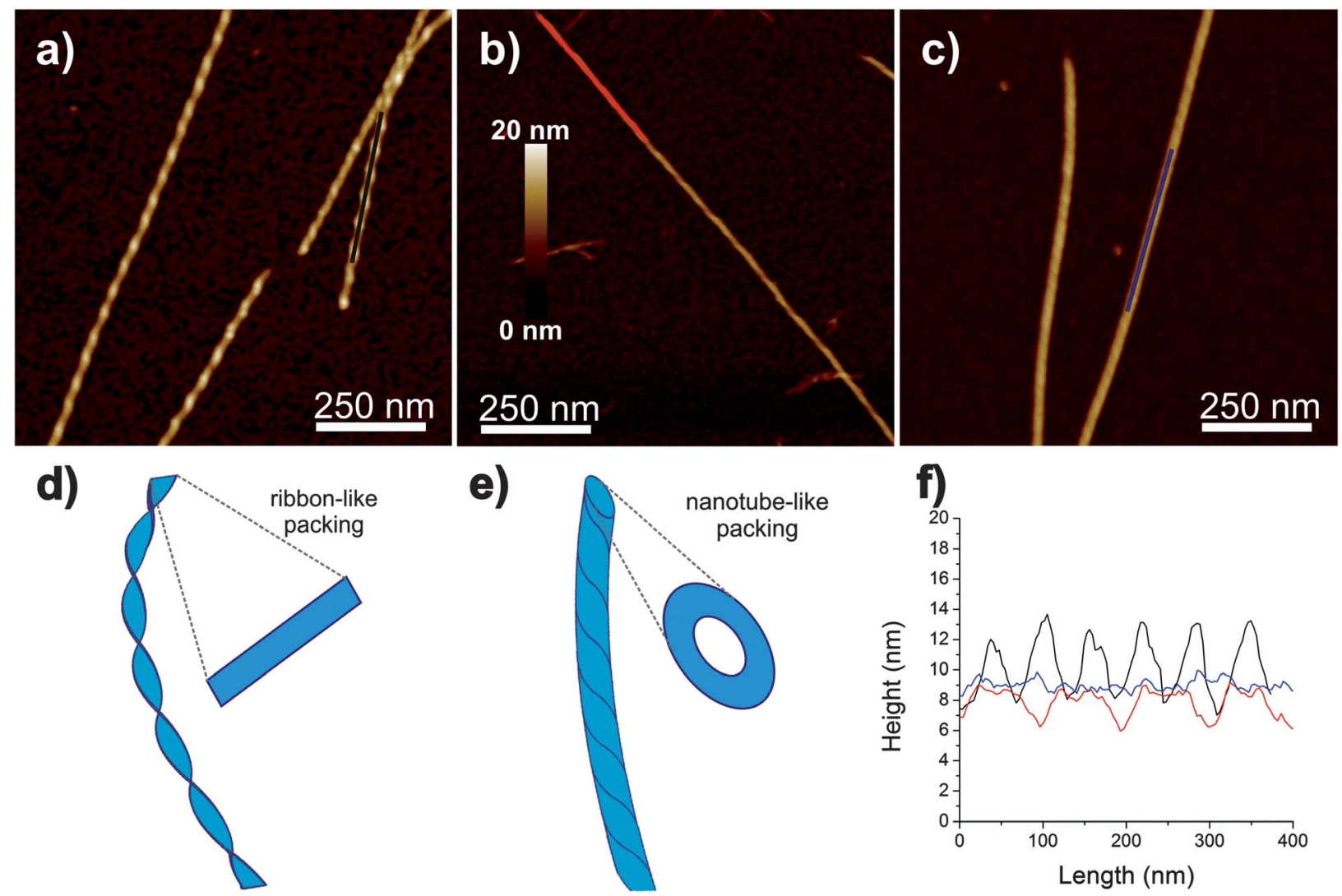

Fig. 1 AFM height images of (a) twisted ribbon, (b) helical ribbon and (c) nanotube-like structures of the end-capped heptapeptide $\mathrm{CH}_{3} \mathrm{CONH}-$ $\beta A \beta A K L V F F-C O N H$. Schematic presentation of (d) twisted ribbon and (e) nanotube-like structures. (f) The height profiles of twisted ribbon (black curve), helical ribbon (red curve) and nanotubes (blue curve). 
measured values of Young's modulus and those extracted by this indirect procedure were in excellent agreement. ${ }^{22}$

This indirectly points to another very important issue in amyloid fibrils, that is, different polymorphic amyloid forms of the same protein or peptide should possess different area moments of inertia, different persistence lengths, but identical Young's moduli. Although this is often assumed in the literature, a direct, experimental evidence has not been given, to the best of our knowledge. In principle, PFQNM should allow for an assessment of this open question, due to the direct measurement of the intrinsic stiffness.

To move forward we need to apply PF-QNM to an amyloid system showing a high level of polymorphic structures. It has been recently shown that the end-capped heptapeptide $\mathrm{CH}_{3} \mathrm{CONH}$ $\beta A \beta A K L V F F-C O N H 2$, modified from the $A \beta(16-20)$ fragment $\mathrm{KLVFF},{ }^{23}$ assembles upon incubation into different morphologies of amyloid fibrils from twisted ribbons to helical ribbons and finally to nanotube-like structures. ${ }^{24}$

Due to the different area moment of inertia $I$ for the three packing schemes we do expect a different persistence length for the three structures, which we estimate (from fibrils with identical height profile and same maximum height), using the bond correlation function $\langle\cos \theta(s)\rangle=\exp \left(-s / 2 l_{\mathrm{p}}\right)$ where $\theta$ is the angle between the tangent vectors to the chain at two points separated by a contour distance $s$ and the factor 2 is used to rescale the exponential decay accounting for the two-dimensional nature of fibrils absorbed on a substrate. ${ }^{32,33}$ This is justified by our recent findings that amyloid fibrils do possess, at large length scales, the fractal exponent characteristic of selfavoiding random walks in $2 \mathrm{D}$ e.g. $3 / 4$, unambiguously demonstrating equilibration of the fibrils in $2 \mathrm{D} .^{27}$ Indeed, in the case of twisted ribbons, the fibrils are more flexible and their persistence length $l_{\mathrm{p}}$ is $3.5 \mu \mathrm{m}$ while for the nanotube-like structures $l_{\mathrm{p}}$ is $18.5 \mu \mathrm{m}$; helical ribbons provide an interesting intermediate case, with $l_{\mathrm{p}}$ of $14.7 \mu \mathrm{m}$. These observations are in perfect agreement with the expected increase in the rigidity of amyloid fibrils showing twisted ribbons $\rightarrow$ nanotubes polymorphism ${ }^{32}$ (Fig. 1).

We then performed PF-QNM experiments to measure the Young's moduli of the modified heptapeptide exhibiting these different morphologies (Fig. 2). In the three cases the measured values are identical within the experimental error: for twisted ribbons the Young's modulus is found to be $2.3 \pm 0.6 \mathrm{GPa}$; for helical ribbons we measure a value of $2.3 \pm 0.7 \mathrm{GPa}$ and for nanotubes $2.4 \pm 0.5 \mathrm{GPa}$. We show in Fig. 2 the analysis of the Young's modulus for the three polymorphic forms twisted ribbons, helical ribbons and nanotubelike structures.
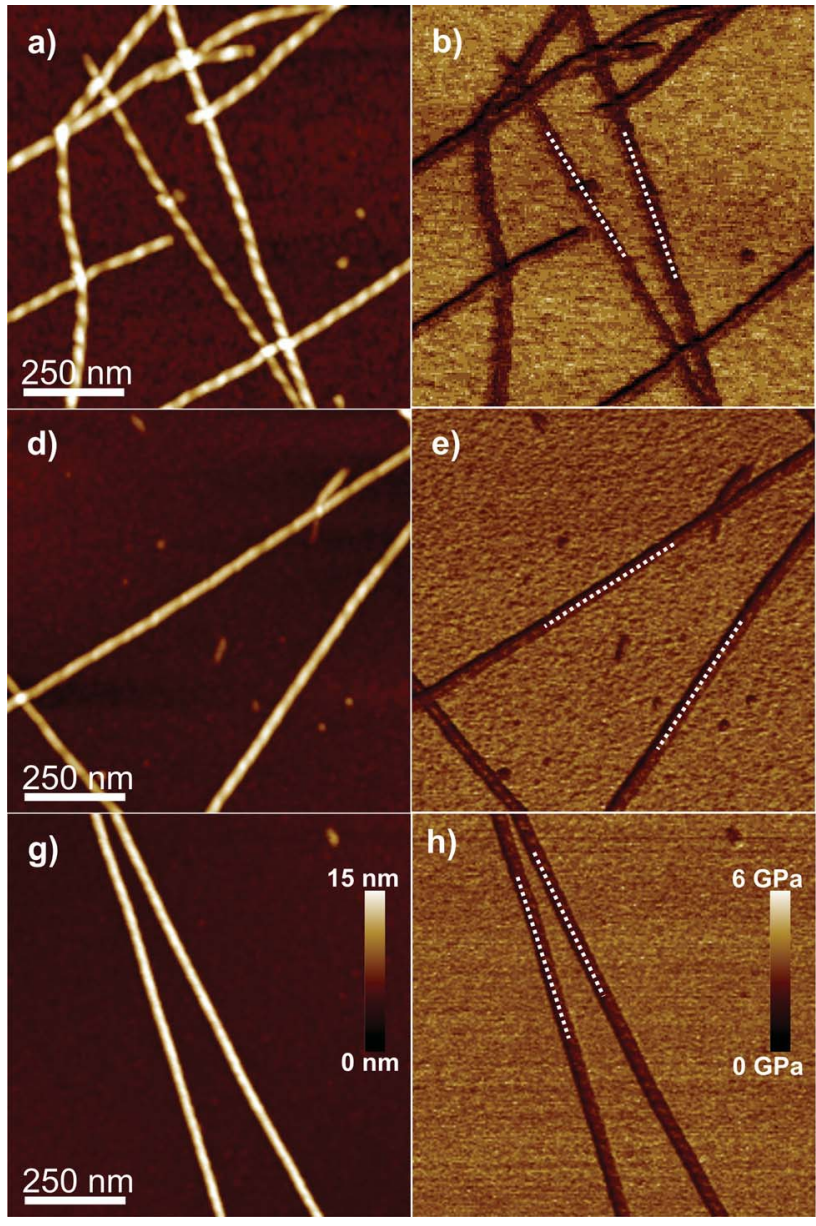
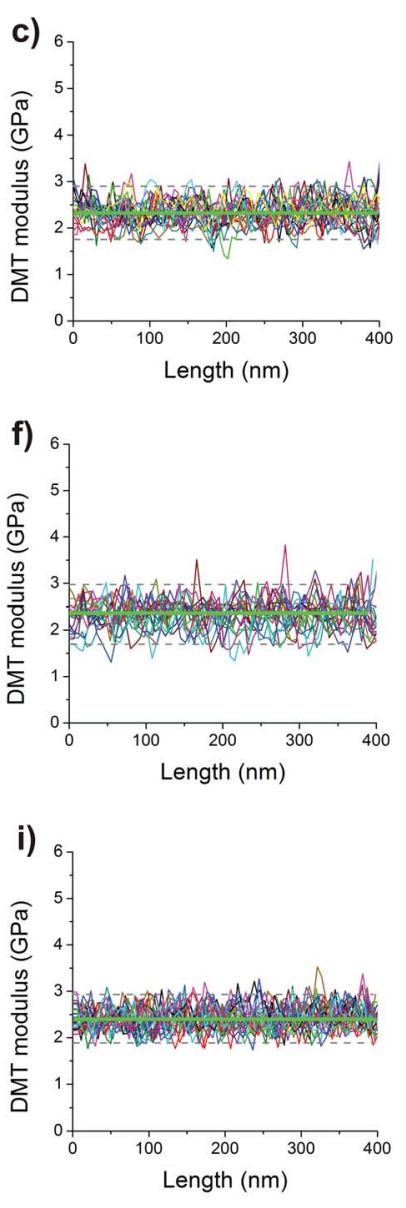

Fig. 2 AFM height image of (a) twisted ribbon, (d) helical ribbon and (g) nanotube-like structures of the end-capped heptapeptide $\mathrm{CH}_{3} \mathrm{CONH}$ $\beta A \beta A K L V F F-C O N H 2$. AFM DMT modulus image of (b) twisted ribbon, (e) helical ribbon and (h) nanotube-like structures. The dashed lines mark the positions at which the DMT modulus was analysed. Profiles of DMT moduli along (c) twisted ribbon, (f) helical ribbon and (i) nanotube-like structures. The data were obtained by analysis of 20 fibrils for each type. 
Table 1 The Young's moduli of several amyloid fibril types measured by the PF-QNM technique

\begin{tabular}{ll}
\hline Amyloid fibrils & Young's modulus \\
\hline $\mathrm{CH}_{3} \mathrm{CONH}-\beta A \beta A K L V F F-C O N H_{2}$ & $2.3 \pm 0.6 \mathrm{GPa}$ \\
Insulin & $3.2 \pm 0.6 \mathrm{GPa}$ \\
$\beta$-Lactoglobulin & $3.7 \pm 0.8 \mathrm{GPa}$ \\
Lysozyme & $2.8 \pm 0.9 \mathrm{GPa}$ \\
Bovine serum albumin & $3.0 \pm 0.6 \mathrm{GPa}$ \\
Ovalbumim & $2.7 \pm 0.8 \mathrm{GPa}$ \\
Tau protein & $3.4 \pm 0.7 \mathrm{GPa}$ \\
$\mathrm{A} \beta(1-42)$ & $3.2 \pm 0.8 \mathrm{GPa}$ \\
$\alpha$-Synuclein & $2.2 \pm 0.6 \mathrm{GPa}$ \\
\hline
\end{tabular}

We have thus demonstrated experimentally by PF-QNM that morphologically different amyloid structures of the same peptide, characterised by a five-fold difference in rigidity, as expressed by the persistence length, do possess identical Young's moduli. These findings have a doubly important relevance: on one hand they offer an important assessment into amyloid fibrils' physical properties; on the other hand they provide an additional solid benchmark for the PFQNM method. Having confirmed that PF-QNM can perfectly disentangle the Young's modulus from topological details of amyloid fibrils, we proceeded to the measurement of the Young's moduli of several different families of amyloid fibrils, assembled from $\alpha$-synuclein, $A \beta(1-42)$, tau protein, insulin, $\beta$-lactoglobulin, lysozyme, ovalbumin and bovine serum albumin fibrils.

The values of the measured Young's moduli are given in Table 1. As a comparison with other available data on Young's modulus of amyloid fibrils, it is worth noting that the value of Young's modulus for insulin amyloid fibrils measured here by PF-QNM is $3.2 \pm$ $0.6 \mathrm{GPa}$, which is very similar to the Young's modulus of insulin reported elsewhere $(3.3 \pm 0.4 \mathrm{GPa}) .{ }^{13}$ More importantly, the values we measure for $\alpha$-synuclein fibrils are nearly identical to the values of Young's modulus independently measured by the PF-QNM by the group of Subramaniam and colleagues. ${ }^{34}$

\section{Conclusions}

By performing PF-QNM experiments on several kinds of mature amyloid fibrils we have shown that the values of the Young's moduli can be entirely disentangled from the overall rigidities associated with different polymorphic forms of the fibrils, and their different area moments of inertia. We have thus demonstrated that the Young's modulus measured by PF-QNM correctly identifies the intrinsic stiffness of the fibril. This has further allowed us to unequivocally conclude that the self-assembly of the same peptide associating into different polymorphic forms of amyloid fibrils with twisted ribbon, helical ribbon and nanotube cross-section always results in the same Young's modulus of the fibril, independent of the specific morphology. We have then measured the Young's moduli of a wide range of amyloid fibril classes and benchmarked, when possible, the measured values with those values available from the literature, showing that all the amyloid classes investigated have Young's moduli in the 2-4 GPa range.

\section{Notes and references}

1 F. Chiti and C. M. Dobson, Annu. Rev. Biochem., 2006, 75, 333-366.

2 R. Nelson, M. R. Sawaya, M. Balbirnie, A. Ø. Madsen, C. Riekel, R. Grothe and D. Eisenberg, Nature, 2005, 435, 773-778.

3 F. Chiti and C. M. Dobson, Nat. Chem. Biol., 2009, 5, 15-22.

4 B. Caughey and P. T. Lansbury, Annu. Rev. Neurosci., 2003, 26, $267-$ 298.

5 C. M. Dobson, Nature, 2003, 426, 884-890.

6 F. Shewmaker, R. P. McGlinchey and R. B. J. Wickner, J. Biol. Chem., 2011, 286, 16533-16540.

7 J. Greenwald and R. Riek, Structure, 2010, 18, 1244-1260.

8 M. R. Chapman, L. S. Robinson, J. S. Pinkner, R. Roth, J. Heuser, M. Hammar, S. Normark and S. J. Hultgren, Science, 2002, 295, $851-855$.

9 E. Gazit, Chem. Soc. Rev., 2007, 36, 1263-1269.

10 I. Cherny and E. Gazit, Angew. Chem., Int. Ed., 2008, 47, 4062-4069.

11 T. P. Knowles and M. J. Buehler, Nat. Nanotechnol., 2011, 6, 469-479.

12 T. P. Knowles, A. W. Fitzpatrick, S. Meehan, H. R. Mott, M. Vendruscolo, C. M. Dobson and M. E. Welland, Science, 2007, 318, 1900-1903.

13 J. F. Smith, T. P. Knowles, C. M. Dobson, C. E. Macphee and M. E. Welland, Proc. Natl. Acad. Sci. U. S. A., 2006, 43, 15806-15811.

14 W. F. Xue, A. L. Hellewell, W. S. Gosal, S. W. Homans, E. W. Hewitt and S. E. Radford, J. Biol. Chem., 2009, 284, 34272-34282.

15 J. Adamcik, J. M. Jung, J. Flakowski, P. De Los Rios, G. Dietler and R. Mezzenga, Nat. Nanotechnol., 2010, 5, 423-428.

16 A. Relini, S. Torrassa, R. Ferrando, R. Rolandi, S. Campioni, F. Chiti and A. Gliozzi, Biophys. J., 2010, 98, 1277-1284.

17 N. Kol, L. Adler-Abramovich, D. Barlam, R. Z. Shneck, E. Gazit and I. Rousso, Nano Lett., 2005, 5, 1343-1346.

18 R. Paparcone, S. Keten and M. J. Buehler, J. Biomech., 2010, 43, 1196-1201.

19 R. Paparcone, S. W. Cranford and M. J. Buehler, Nanoscale, 2011, 3, $1748-1755$.

20 M. Solar and M. J. Buehler, Nanoscale, 2012, 4, 1177-1183.

21 R. Paparcone and M. J. Buehler, Biomaterials, 2011, 32, 3367-3374.

22 J. Adamcik, A. Berquand and R. Mezzenga, Appl. Phys. Lett., 2011, 98, 193701.

23 V. Castelletto, I. W. Hamley, Ç. Cenker, U. Olsson, J. Adamcik, R. Mezzenga, J. F. Miravet, B. Escuder and F. Rodríguez-Llansola, J. Phys. Chem. B, 2011, 115, 2107-2116.

24 J. Adamcik, V. Castelletto, S. Bolisetty, I. W. Hamley and R. Mezzenga, Angew. Chem., Int. Ed., 2011, 50, 5495-5498.

25 J. M. Jung, G. Savin, M. Pouzot, C. Schmitt and R. Mezzenga, Biomacromolecules, 2008, 9, 2477-2486.

26 C. Lara, J. Adamcik, S. Jordens and R. Mezzenga, Biomacromolecules, 2011, 12, 1868-1875.

27 C. Lara, I. Usov, J. Adamcik and R. Mezzenga, Phys. Rev. Lett., 2011, 107, 238101.

28 A. Jan, D. M. Hartley and H. A. Lashuel, Nat. Protoc., 2010, 5, 11861209.

29 L. Giehm, N. Lorenzen and D. E. Otzen, Methods, 2011, 53, 295-305.

30 S. Wegmann, Y. J. Jung, S. Chinnathambi, E. M. Mandelkow, E. Mandelkow and D. J. Muller, J. Biol. Chem., 2010, 285, 2730227313.

31 J. Adamcik, F. Valle, G. Witz, K. Rechendorff and G. Dietler, Nanotechnology, 2008, 19, 384016.

32 J. Adamcik and R. Mezzenga, Macromolecules, 2012, 45, 1137-1150.

33 F. Valle, M. Favre, P. De Los Rios, A. Rosa and G. Dietler, Phys. Rev. Lett., 2005, 95, 158105.

34 K. Sweers, K. van der Werf, M. Bennink and V. Subramaniam, Nanoscale Res. Lett., 2011, 6, 270. 